\title{
Analysis the Curative Effect of One-Third Steel Plate Applied in the Medial Column of Pilon Fracture
}

\section{Zhuo Wang (D396346343@qq.com)}

Northern Jiangsu People's Hospital

\section{Research article}

Keywords: Ruedi-Augower, Radiology

Posted Date: November 9th, 2020

DOI: https://doi.org/10.21203/rs.3.rs-102432/v1

License: (1) This work is licensed under a Creative Commons Attribution 4.0 International License. Read Full License 


\section{Abstract}

Objective: To analysis the difference of efficacy between the one-third tube steel plate and screw on the medial column of Ruedi-Augower III pilon fracture.

Methods: The clinical data of 26 patients with Ruedi-Augower type III pilon fractures admitted from April 2016 to April 2019 were collected. Patients were divided into two groups according to the reconstruction materials. In the control group, 16 cases were fixed with screws on the medial column. In the observation group, 10 cases were reconstructed with a one-third tube steel plate on the medial column. The reduction quality was evaluated by using Burwell-Charnley's radiographic criteria. Ankle function was evaluated by using the American Orthopedic Foot and Ankle Society (AOFAS) ankle-hindfoot score. The preoperative swelling time, operation time, intraoperative blood loss, fracture healing time, postoperative weight-bearing time, postoperative AOFAS anklehindfoot score and postoperative Burwell-Charley Radiology Score were compared between the two groups.

Results: In the all patients, nobody developed deep tissue infections. The fracture healing time was $15.07 \pm 0.93$ weeks, and the weight-bearing time after the operation was $6.91 \pm 0.58$ weeks. The satisfactory rate was $90.00 \%$ according to Burwell-Charnley's reduction criteria and the second month postoperative AOFAS ankle-hindfoot score was $66.30 \pm 2.79$. As for the control group, the fracture healing time was $15.83 \pm 0.56$ weeks, and the postoperative weight-bearing time was $8.60 \pm 0.50$ weeks. Only $70 \%$ fractures accomplished anatomic reduction on the basis of Burwell-Charnley's reduction criteria and the AOFAS ankle-hindfoot score only got $75.80 \pm 1.94$ in the second month after surgery.

Conclusion: When Ruedi-Augower type III pilon fractures were treated with one-third tube steel plate in the medial column, compared with the simple screw fixation, it took into account the excellent healing of soft tissues meanwhile it promised the weight-bearing time earlier and less loss rate of long-term postoperative reduction.

\section{Introduction}

Tibia fracture is the most common fracture of all long bone fractures and Pilon fractures account for $5-7 \%{ }^{[1]}$ of tibia fractures. With more and more accidents such as high-energy trauma and car accidents occurring in recent years, the incidence of pilon fractures grows with each passing day. Pilon fracture was first defined by a radiologist. He compared the distal tibia to a medical pestle which lead to distal tibia fractures by hitting the talus vertically and pushing the talus into the distal tibia. It was an special type of ankle fractures involving severe comminution of the articular surface of the distal tibial, bone loss ${ }^{[2]}$,compression of cancellous bone and fibula fracture and mostly caused by the combined forces of axial compression, shear, and rotation. Soft tissue swelling, severe damage to the articular surface, high incidence of postoperative infection, and nonunion of fractures all bring huge difficulties to the treatment and prognosis of Ruedi-Augower type III pilon fractures. In order to achieve anatomical reduction and accomplish early functional exercise, the choice of effective internal fixation is particularly important ${ }^{[3]}$. At present, regarding the surgical treatment of pilon fractures, there are different opinions on the fixation of the medial column. Based on the principle of direct access to the fracture site during the operation, reducing the surgical incision as much as possible, and reducing the peeling of soft tissue and periosteum, clinicians use screws, clover plate and traditional reconstruction plate to open reduction and internal fixation. By collecting case data and consulting literature, the author found that choosing screws to reconstruct the medial column can reduce the irritation to the thin soft tissue to a certain extent, cause little 
damage to the blood supply of the distal tibia and be more friendly to the bone tissue regeneration after surgery. However, the fixing strength is not enough so the postoperative weight-bearing time will be extended. Long-term bedridden patients not only have to face the complications such as joint stiffness and postoperative adhesion, but also bear the high incidence of respiratory and urinary infections. When clover plate and reconstruction plate were adopted, although the strong fixation ensures the patient's early functional exercise and reduces the possibility of postoperative reduction loss, the thick plate brings huge challenges to fracture healing and soft tissue recovery. Ruedi-Augower type III pilon fractures usually accompanies by comminuted fracture and complex fracture lines, which increase the difficulty of the operation especially by adopting the clover plate and reconstruction plate. This article retrospectively analyzed the clinical data of pilon patients treated with the two internal fixation methods in our hospital to explore more effective fixation methods and obtain better cure effects.

\section{Patients And Methods}

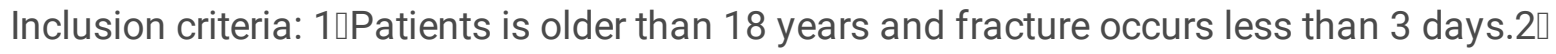

Patients were diagnosed with Ruedi-Augower III pilon fracture after X-ray or CT examination.3๓Without other severe fractures including calcaneus and talus.4ロPatients were of normal function before fractures occurred without congenital ankle deformity.5ロAt least half one year follow up.

Exclusion criteria:1 1 lrreversible vascular and nerve damage in the ankle joint.2₫Pathological fracture.3ロThe patient's basic condition does not allow surgery.

The ethics committee of Subei People's Hospital of Jiangsu Province approved the present study. Twenty-six pilon (AO/OTA 43-C3) fractures treated between April 2016 and April 2019 were retrospectively reviewed. According to different fixation methods, they were divided into control group and observation group. The control group chose screw, and the observation group chose one-third tube steel plate. All patients completed debridement within 8 hours of admission, and all patients received calcaneal traction in the emergency room. The affected limb was raised and immobilized, and when the local swelling was slightly relieved with subtle skin folds visible, the tension blisters subsided. We will synthesize blood biochemical indicators and perform internal fixation surgery ${ }^{[4]}$.

Intraspinal anesthesia would be taken in the surgery and patients would be taken a "floating position" with a thigh pneumatic tourniquet. The limb was sterilized from the level of the tourniquet to the toes. The posterolateral approach was taken between the outer edge of the affected Achilles tendon and the fibula, taking care of the injury of sural nerve and small saphenous vein. Then fully expose the fibula by pulling the outer peroneus brevis tendon and the inner flexor longus tendon to correct fibula fracture rotation deformity. When the reduction became satisfactory, the lateral malleolus plate would be applied. Next, the fibula long and brevis muscle was separated Bluntly to expose the posterior column fracture. The reduction of the posterior Volkmann fracture block was used as a sign of the reduction of the posterior tibia cortex. C-arm machine was used multidirectional and repeated to ensure that the posterior column was anatomically reduced and fixed with a Tshaped plate. Then the patient takes a supine position and keeps the ankle joint toe flexion. The anterior median incision was taken the between the tibial crest and the tibial anterior muscle. The incision length was determined according to length of the medial malleolus fracture. We should keep a distance of more than $7 \mathrm{~cm}$ between the 
incisions. The skin and subcutaneous soft tissue were sequentially incised to fully expose the anterior and medial sides of the distal tibia, taking care to prevent damage to the saphenous nerve and saphenous vein. First of all, the impacted "die-punch" bones should be reduced. Then clean the congestion on the articular surface and the embedded soft tissue. Tillaux-Chaput nodules was used to accurately determine the level of the ankle joint surface, so as to reposition the ankle joint surface and ensure the smoothness of the distal tibia joint surface as much as possible. Bone graft should be used to fill osseous voids during fracture fixation to substitute for bone loss. After the results of fluoroscopy were satisfied, the anterolateral L-shaped locking plate was used for fixation. Finally, reduce the medial malleolus under the same incision. Observation group: The medial column was temporarily fixed with Kirschner wire when the fluoroscopy was assessed as anatomic reduction, a one-third tubular steel plate would be fixed firmly. The fractures with osteoporosis should be operated as slightly as possible to avoid further fractures. The screws were fixed after reduction, and the screws were placed perpendicularly to the fracture line. The arrangement of the screws was as dispersed as possible, meanwhile, the screws should be avoided from inserting into the ankle joint. Postoperatively, A large amount of normal saline is used to flush the operation area repeatedly, close the incision layer by layer, and wrap it with sterile gauze.

The patients were routinely treated with anti-inflammatory, labor pains, anti-infection and change the dressing regularly. All patients were injected subcutaneously with $4000 \mathrm{U}$ low molecular weight heparin 24 hours later to prevent deep vein thrombosis. On the second day, under the guidance of the rehabilitation department, routinely performed flexion and extension exercises for the knees, ankles and toes. The effect of surgical reduction and the imaging scores would be assessed by fluoroscopy. Sutures were removed 2 weeks after the routine operation, re-examination in 4 weeks, and partial weight bearing on crutches was carried out in the 6th to 8th weeks as appropriate.

\section{Results}

Patients in two groups successfully completed the internal fixation treatment, and all patients' incisions healed well without postoperative neurological complications. 


\begin{tabular}{|c|c|c|c|c|c|c|c|}
\hline Groups & Age & $\begin{array}{l}\text { Male/ } \\
\text { Female }\end{array}$ & $\begin{array}{l}\text { Swelling } \\
\text { time } \\
\text { before } \\
\text { surgery } \\
\text { (day) }\end{array}$ & $\begin{array}{l}\text { Operation } \\
\text { time } \\
\text { (h) }\end{array}$ & $\begin{array}{l}\text { Intraoperative } \\
\text { blood } \\
\text { loss }(\mathrm{ml})\end{array}$ & $\begin{array}{l}\text { Fracture } \\
\text { Healing } \\
\text { time(week) }\end{array}$ & $\begin{array}{l}\text { Weight-bearing } \\
\text { time after } \\
\text { operation(week) }\end{array}$ \\
\hline $\begin{array}{l}\text { Observation } \\
\text { group }(\mathrm{N}= \\
10)\end{array}$ & $\begin{array}{l}55.20 \\
\pm \\
10.10\end{array}$ & $6 / 4$ & $\begin{array}{l}10.20 \pm \\
4.05\end{array}$ & $\begin{array}{l}2.83 \pm \\
0.47\end{array}$ & $120 \pm 48.30$ & $\begin{array}{l}15.07 \pm \\
0.98\end{array}$ & $6.91 \pm 0.61$ \\
\hline $\begin{array}{l}\text { Control } \\
\text { group }(\mathrm{N}= \\
16)\end{array}$ & $\begin{array}{l}48.7 \\
\pm \\
10.30\end{array}$ & $11 / 5$ & $\begin{array}{l}10.50 \pm \\
3.69\end{array}$ & $\begin{array}{l}2.17 \pm \\
0.45\end{array}$ & $131 \pm 84.00$ & $\begin{array}{l}15.84 \pm \\
0.59\end{array}$ & $8.60 \pm 0.53$ \\
\hline$t / x^{2}$ & 1.39 & 2.40 & -0.17 & 1.96 & -0.36 & -2.13 & -6.63 \\
\hline$P$ & 0.18 & 0.12 & 0.86 & 0.07 & 0.72 & 0.05 & $\varangle 0.01$ \\
\hline \multicolumn{2}{|l|}{ Groups } & \multicolumn{3}{|c|}{$\begin{array}{l}\text { Burwell-Charley Radiology Score } \\
\text { excellent good general }\end{array}$} & \multicolumn{2}{|c|}{ In total Excellent rate } & \\
\hline \multicolumn{2}{|c|}{$\begin{array}{l}\text { Observation group }(\mathrm{N} \\
=10)\end{array}$} & 10 & 0 & & \multicolumn{2}{|c|}{$10100 \%$} & \\
\hline \multicolumn{2}{|c|}{$\begin{array}{l}\text { Control group }(\mathrm{N}= \\
\text { 16) }\end{array}$} & 10 & 2 & & \multicolumn{2}{|c|}{$1660 \%$} & \\
\hline \multicolumn{2}{|l|}{$t / X^{2}$} & 5.00 & & & & & \\
\hline \multicolumn{2}{|l|}{$P$} & 0.03 & & & & & \\
\hline
\end{tabular}

\begin{tabular}{|lllll|}
\hline Groups & & \multicolumn{3}{c|}{ AOFAS ankle-hindfoot score } \\
\hline month & First month & Second month & Third month & Sixth month \\
\hline Observation group $(\mathrm{N}=10)$ & $65.50 \pm 2.01$ & $75.80 \pm 2.04$ & $86.00 \pm 2.26$ & $86.20 \pm 1.69$ \\
\hline Control group $(\mathrm{N}=16)$ & $56.40 \pm 3.53$ & $66.30 \pm 2.95$ & $73.90 \pm 3.11$ & $85.00 \pm 0.94$ \\
\hline $\mathrm{t} / \mathrm{X}^{2}$ & 7.08 & 8.38 & 9.96 & 1.96 \\
\hline $\mathrm{P}$ & 0.00 & 0.000 & 0.000 & 0.07 \\
\hline
\end{tabular}

Ten patients were treated with plates, while sixteen patients were treated with screw. All patients underwent calcaneal traction to assist with swelling and reduction before operation. Sixteen patients were treated with screws, while ten patients were treated with plates.Patients averaged 3.2 surgical procedures (range 2-8) for definitive fixation, soft tissue coverage, and planned bone grafting. If a deep wound infection or nonunion developed, then an average of 2.8 (range 2-3) additional surgical procedures were required. Eighteen of the open wounds

were closed either primarily or with split-thickness skin graft (grades I-IIIA), while ten patients (grade IIIB) required flap coverage (five fasciocutaneous or pedicle muscle flaps and five free tissue transfers). One patient 
had a partial flap loss requiring revision coverage surgery.

Functional outcome questionnaires were completed by 26 patients. AOFAS ankle-hindfoot score and BurwellCharley Radiology Score were compared between two groups. The weight-bearing time after operation of the observation groups 6.91 weeks, which compared favorably to the control groups. Of the twenty-six patients completing outcome questionnaires, all had returned to full weight bearing with 4 having pain with weight bearing in the control group and 1 having pain with weight bearing in the observation group. Four patients required job modification from pre-injury employment in the control group.

\section{Discussion}

Pilon fractures have been defined since 1911, and there are endless classification methods. For example, the $\mathrm{V}$ type mentioned by Lauger-Hansen in 1963, was a longitudinal compression type. The Ruedi-Allgower and AO/OTA classification are widely used in clinical diagnosis. Both classifications have a certain effect on the pilon fractures, such as the degree of joint comminution and displacement. With the improvement of technology and cognition, Klammer ${ }^{[7]}$ and others proposed that the diagnosis, treatment and postoperative evaluation of pilon fractures should be based on CT three-dimensional reconstruction. Subsequently, Tang Xin ${ }^{[8]}$ proposed the four-column theory of fracture classification based on CT three-dimensional reconstruction. Compared with the classic classification, this theory is more instructive in the selection of clinical surgical incisions and the internal fixation materials.

The four-column classification refers to dividing the inner and outer malleolus into anterior and posterior columns by the line connecting the vertices of the medial and lateral malleolus, and dividing them into medial and lateral columns by the central axis of the sagittal plane of the distal articular surface of the tibia and fibula. There are many ways to fix the medial column, but improper treatment and insecure fixation often cause skin necrosis, failure of internal fixation, traumatic arthritis, and joint dysfunction. As we all know, the cross section of the distal tibia moves from a triangle to a quadrilateral from top to bottom, and the soft tissue coverage of the tibia becomes less and less. However two thirds of the tibia's blood supply comes from intramedullary vessels, and one third comes from the soft tissue attached to the surface.Pilon fractures usually cause intramedullary vascular damage, and Open reduction and internal fixation aggravated the damage of soft tissues and further reduced the blood supply to the distal tibia, leading to non-union, infection, resulting in post-traumatic arthritis and other complications. Kottmeier ${ }^{[9]}$ also found that compared with other fractures, the incidence of nonunion of the distal tibia after surgery is higher. At the same time, Blauth ${ }^{[10]}$ and others proposed the 3P principle in the treatment of pilon fractures. The first point is to protect the blood supply of bone and tissue. Falzarano ${ }^{[11]}$ and others were worried that the medial malleolus itself had less soft tissue and high skin tension. So Kirschner wires, lag screws, hollow nails and other internal fixation materials with less soft tissue peeling were adopted in the fixation. This method avoids the high incidence of complications such as skin necrosis and nonunion caused by internal plants. Compared with the medial column reconstructed by the steel plate, the operation time, blood loss, surgical incision and material cost are reduced, and a good effect has been achieved to a certain

extent. However, during the actual operation, Tong ${ }^{[12]}$ found that due to the comminution of the distal tibia, especially the comminution of the lateral cortex of the distal tibia,which makes the proximal ends of lag screws, hollow nails and Kirschner wires relatively loose. At the same time, the author found that the internal bone mass also moved slightly during the flexion and extension of the ankle joint. Jonathan R. Danoff ${ }^{[13]}$ also used 
bicortical screws to fix the medial column at an angle. After the operation, it was found that although the number of infected patients was greatly reduced, the postoperative weight-bearing pain rate was as high as $11 \%$, and $25 \%$ of patients had to change jobs because of postoperative ankle function decline. Although screw fixation reduces the possibility of postoperative skin necrosis, bone exposure, non-union of fractures and delayed union, but also because the patient is late in the ground, functional exercise is delayed, and lung infection, pressure sores, joint stiffness and ankle joint function decreased ${ }^{[14]}$. Early landing may bring about undesirable consequences such as loss of postoperative reduction, breakage of internal fixation, and re-fracture. Based on the unique physiological structure and weight-bearing function of the ankle joint, a single metal screw or Kirschner wire is more difficult to resist shear force during early functional exercise. Therefore, more and more clinicians are considering the use of strong fixation materials for the medial column that are thinner and less irritating to soft tissues. Hong Gao ${ }^{[15]}$ and others used a multiaxial locking plate to fix the medial column and found that although the plate was thicker than the screw, the occurrence of soft tissue infections was not as high as expected. Feng $\mathrm{Ku}^{[16]}$ and Amorosa ${ }^{[17]}$ used tubular steel plate and locking plate respectively and achieved certain curative effects in the reconstruction of the medial column. Adam M. Wegner ${ }^{[18]}$ and others also confirmed that for pilon medial column oblique fractures, the rigidity of the anti-slide plate fixation is four times that of the single cortical fixation screw. By comparing the X-ray and postoperative AOFAS ankle function scores of the observation group and the control group after 3 months and later follow-up, the control group performed early postoperative landing and functional exercises, and some patients suffered loss of postoperative medial column reduction three months after surgery. As for the observation group, due to the strong fixation of the medial column the safety of early weight-bearing activities was ensured. At the same time, BrianAUthgenannt ${ }^{[19]}$ and others also found that under the premise of firm internal fixation, partial load-bearing in the early stage has a better effect on the fracture healing. By assessing the postoperative AOFAS ankle function score, the AOFAS ankle function scores in the observation group in the first, second and third months after the operation were significantly higher than those in the control group, which further indicated that for the reconstruction of the medial column, one-third of the tubular steel plate was more stable than the screw. During the postoperative follow-up, all patients in the observation group had no adverse complications such as soft tissue irritation, infection, and bone nonunion. Li Jianjun, Zhang Hongbin ${ }^{[20]}$ and others analyzed from Tang Xin's four-pillar theory, axial impact force causes the talus to shift inside the ankle joint anterior and lateral, causing the anterolateral and central area of the distal tibial articular surface to be affected. As the injury increases, the ankle joint Varus and the medial column injury is more serious than the lateral column. And JustinM ${ }^{[21]}$ also found that the proper fixation of the medial column reduces the risk of nonunion after pilon fracture. Based on the principle of early functional exercise, the strong fixation of the medial column becomes more and more important.

Due to the limitations of time and conditions, our follow-up only lasted for half a year. The long-term complications of all patients may not be fully understood. Locking plates and reconstruction plates were not included in the study, and the parallel control group was not set enough. Small sample size brings bias to research. Furthermore, using one-third of the tubular steel plate is economically more expensive than the screw set. However, comprehensively, the author believes that for the reconstruction of the medial column of pilon fractures, one-third of the tubular steel plate has the characteristics of stronger fixation and less soft tissue irritation. The more stable medial column supports each other with the other three columns to form a stronger 
ankle joint, which ensures the patient's early functional exercise and is more conducive to the fracture healing of the patient. It is the preferred material for the fixation of the medial column of pilon fractures.

\section{Abbreviations}

AFOAS: American Orthopedic Foot Andankle Society

\section{Declarations}

Ethics approval and consent to participate冈Dear editor I will send to you later

Consent for publication $₫$ Agree

Availability of data and materials $₫$ Available

Competing interests $₫$ Not applicable

Funding: Not applicable

Authors' contributions: Not applicable

Acknowledgements: Not applicable

Authors' information (optional): Wang Zhuo, Northern Jiangsu People's Hospital, China, E-

mail:3963463343@qq.com

\section{References}

1. Chen $\mathrm{H}$, Cui X, Ma B. Staged procedure protocol based on the four-column concept in the treatment of AO/OTA type 43-C3.3 pilon fractures. J Int Med Res. 2019 May;47(5):2045-55. Doi:

10.1177/0300060519836512. Epub 2019 Mar 19. PMID: 30890008; PMCID: PMC6567754.

2. McCann PA, Jackson M, Mitchell ST. Complications of definitive open reduction and internal fixation of pilon fractures of the distal tibia. Int Orthop. 2011 Mar;35(3):413-8. Doi:10.1007/s00264-010-1005-9. Epub 2010 Mar 30.PMID: 20352430; PMCID: PMC3047643.

3. Stufkens SA, van den Bekerom MP, Kerkhoffs GM. Long-term outcome after 1822 operatively treated ankle fractures: a systematic review of the literature. Injury. 2011 Feb;42(2):119 - 27.

doi:10.1016/j.injury.2010.04.006. PMID: 20444447.

4. Gardner MJ, Mehta S, Barei DP. Treatment protocol for open AO/OTA type C3 pilon fractures with segmental bone loss. J Orthop Trauma. 2008Aug;22(7):451-7. Doi: 10.1097/BOT.0b013e318176b8d9. PMID: 18670284.

5. Burwell HN, Charnley AD. The treatment of displaced fractures at the ankle by rigid internal fixation and early joint movement. J Bone Joint Surg Br. 1965Nov;47(4):634-60. PMID: 5846764.

6. Kaikkonen A, Kannus P, Järvinen M. A performance test protocol and scoring scale for the evaluation of ankle injuries. Am J Sports Med. 1994 Jul-Aug;22(4):462-9. Doi: 10.1177/036354659402200405. PMID: 7943510.

7. Klammer G, Kadakia AR, Joos DA. Posterior pilon fractures: a retrospective case series and proposed classification system. Foot Ankle Int. 2013 Feb;34(2):189-99. PMID: 23413057.

8. Tang Xin T, Peifu Lv, Decheng, et al.. Pilon fractures: a new classification and therapeutic strategies. Chin Med J (Engl). 2012;125:2487-92. 
9. Kottmeier SA, Madison RD, Divaris N. Pilon Fracture: Preventing Complications. J Am Acad Orthop Surg. 2018 Sep 15;26(18):640-651. doi:10.5435/JAAOS-D-17-00160. PMID: 30134307.

10. Blauth M, Bastian L, Krettek C. Surgical options for the treatment of severe tibial pilon fractures: a study of three techniques. J Orthop Trauma. 2001 Mar-Apr;15(3):153-60. Doi:10.1097/00005131-20010300000002.PMID: 11265004.

11. Falzarano G, Pica G, Medici A. Foot Loading and Gait Analysis Evaluation of Nonarticular Tibial Pilon Fracture: A Comparison of Three Surgical Techniques. J Foot Ankle Surg.2018 Sep-Oct;57(5):894-898. Doi: 10.1053/j.jfas.2018.03.025. Epub 2018 Jun 15.PMID: 29914730.

12. Tong $\mathrm{D}$, Ji F, Zhang $\mathrm{H}$. Two-stage procedure protocol for minimally invasive plate osteosynthesis technique in the treatment of the complex pilon fracture. Int Orthop. 2012 Apr;36(4):833-7. doi:10.1007/s00264-0111434-0. Epub 2011 Dec 20. PMID: 22183151; PMCID: PMC3311799.

13. Danoff JR, Saifi C, Goodspeed DC. Outcome of 28 open pilon fractures with injury severity-based fixation. Eur J Orthop Surg Traumatol. 2015 Apr;25(3):569 - 75. Doi: 10.1007/s00590-014-1552-7. Epub 2014 Sep 26. PMID:25256799.

14. Cui $X$, Chen $H$, Rui Y. Two-stage open reduction and internal fixation versus limited internal fixation combined with external fixation: a meta-analysis of postoperative complications in patients with severe Pilon fractures. J Int Med Res. 2018 Jul;46(7):2525-2536. doi:10.1177/0300060518776099. Epub 2018 Jun 19. PMID: 29916291; PMCID: PMC6124300.

15. Gao H, Zhang CQ, Luo CF. Fractures of the distal tibia treated with polyaxial locking plating. Clin Orthop Relat Res. 2009 Mar;467(3):831-7. Doi:10.1007/s11999-008-0459-1. Epub 2008 Aug 22. PMID:18719970; PMCID: PMC2635458.

16. Feng Kua C, Chuanjie, Li D, et al, ç】. A clinical study of 28 cases of serious fractures of the medial malleolus treated with tubular steel plate internal fixation [J]. Chinese Journal of Bone and Joint Injury,2017,32(12):1323-1324. DOI:10.7531/j.issn.1672-9935.2017.12.035.

17. Amorosa LF, Brown GD, Greisberg J. A surgical approach to posterior pilon fractures. J Orthop Trauma. 2010 Mar;24(3):188 - 93. doi:10.1097/BOT.0b013e3181b91927. PMID: 20182256.

18. Wegner AM, Wolinsky PR, Robbins MA. Antiglide plating of vertical medial malleolus fractures provides stiffer initial fixation than bicortical or unicortical screw fixation. Clin Biomech (Bristol, Avon). 2016 Jan;31:29-32. Doi: 10.1016/j.clinbiomech.2015.10.005. Epub 2015 Oct 16. PMID: 26482240.

19. Uthgenannt BA, Kramer MH, Hwu JA. Skeletal self-repair: stress fracture healing by rapid formation and densification of woven bone. J Bone Miner Res. 2007 Oct;22(10):1548-56. doi:10.1359/jbmr.0070614. PMID:17576168; PMCID: PMC3680519.

20. Li jianjun, Hongbin Z. Wu zhixin et al. Comparative study of internal and external column injury of Pilon fracture by stage open reduction and internal fixation [J]. Chinese Journal of Bone and Joint Injury,2016,31(4):432-434. DOI:10.7531/j.issn.1672-9935.2016.04.037.

21. Haller JM, Holt D, Rothberg DL, Kubiak EN, Higgins TF. Does early versus delayed spanning external fixation impact complication rates for high-energy tibial plateau and plafond fractures? Clin Orthop Relat Res. 2016;474(6):1436-44.

\section{Figures}



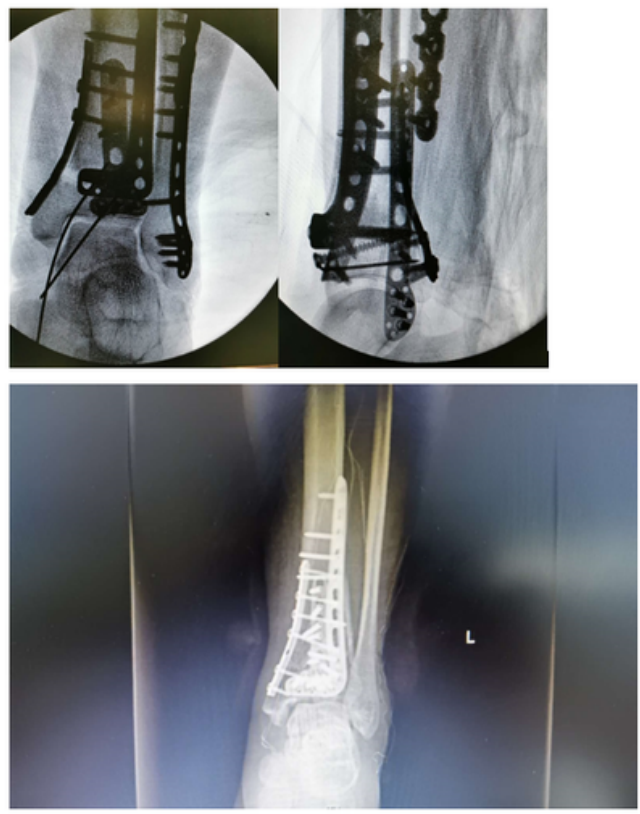
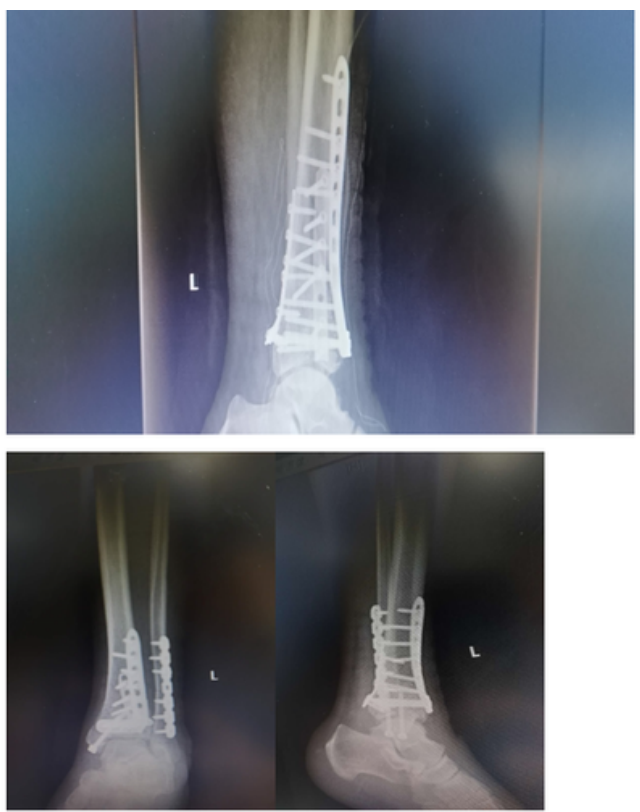
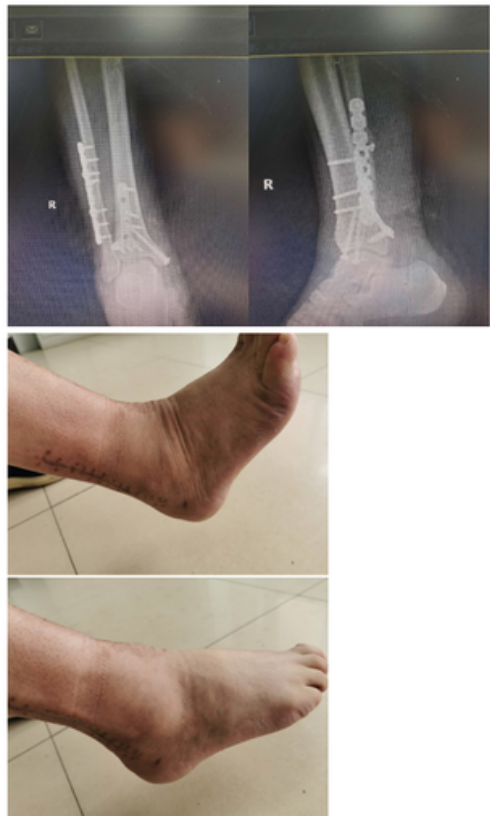

\section{Figure 1}

AOFAS ankle-hindfoot score and Burwell-Charley Radiology Score were compared between two groups. The weight-bearing time after operation of the observation groups 6.91 weeks, which compared favorably to the control groups. Of the twenty-six patients completing outcome questionnaires, all had returned to full weight bearing with 4 having pain with weight bearing in the control group and 1 having pain with
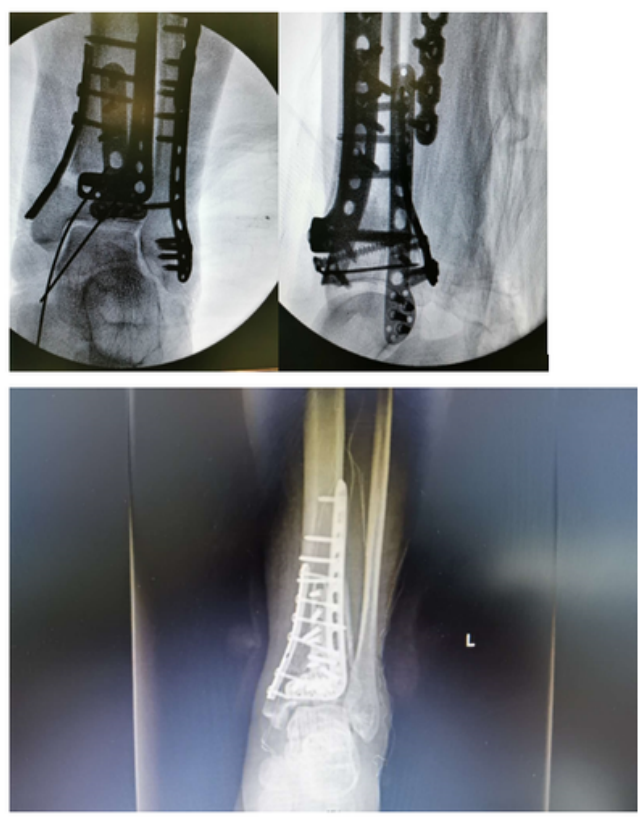
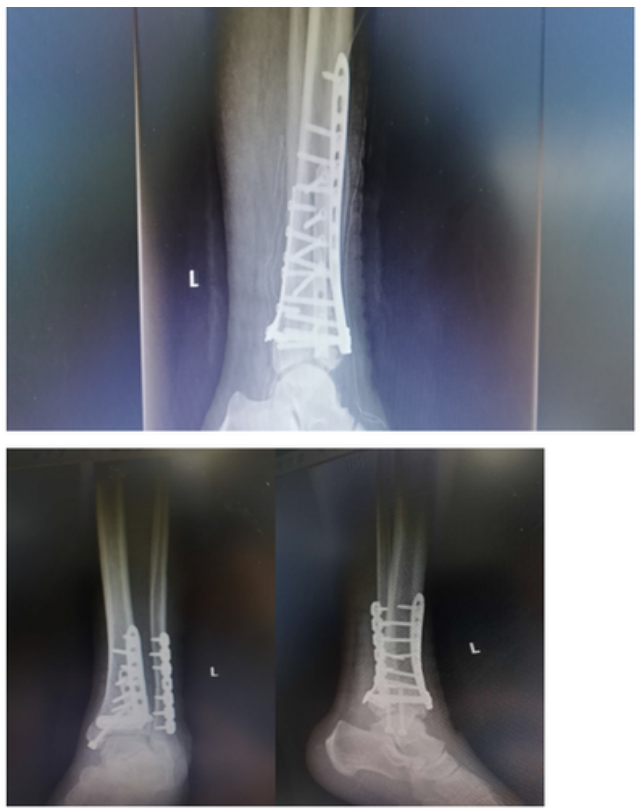

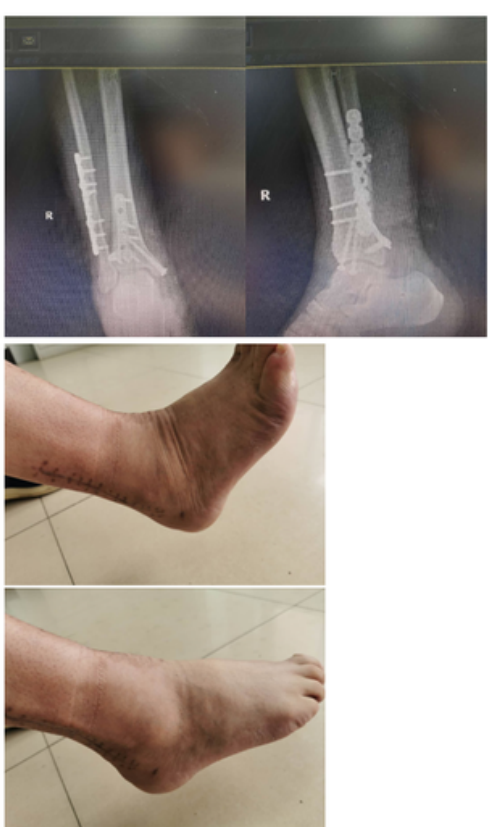

\section{Figure 1}

AOFAS ankle-hindfoot score and Burwell-Charley Radiology Score were compared between two groups. The weight-bearing time after operation of the observation groups 6.91 weeks, which compared favorably to the 
control groups. Of the twenty-six patients completing outcome questionnaires, all had returned to full weight bearing with 4 having pain with weight bearing in the control group and 1 having pain with
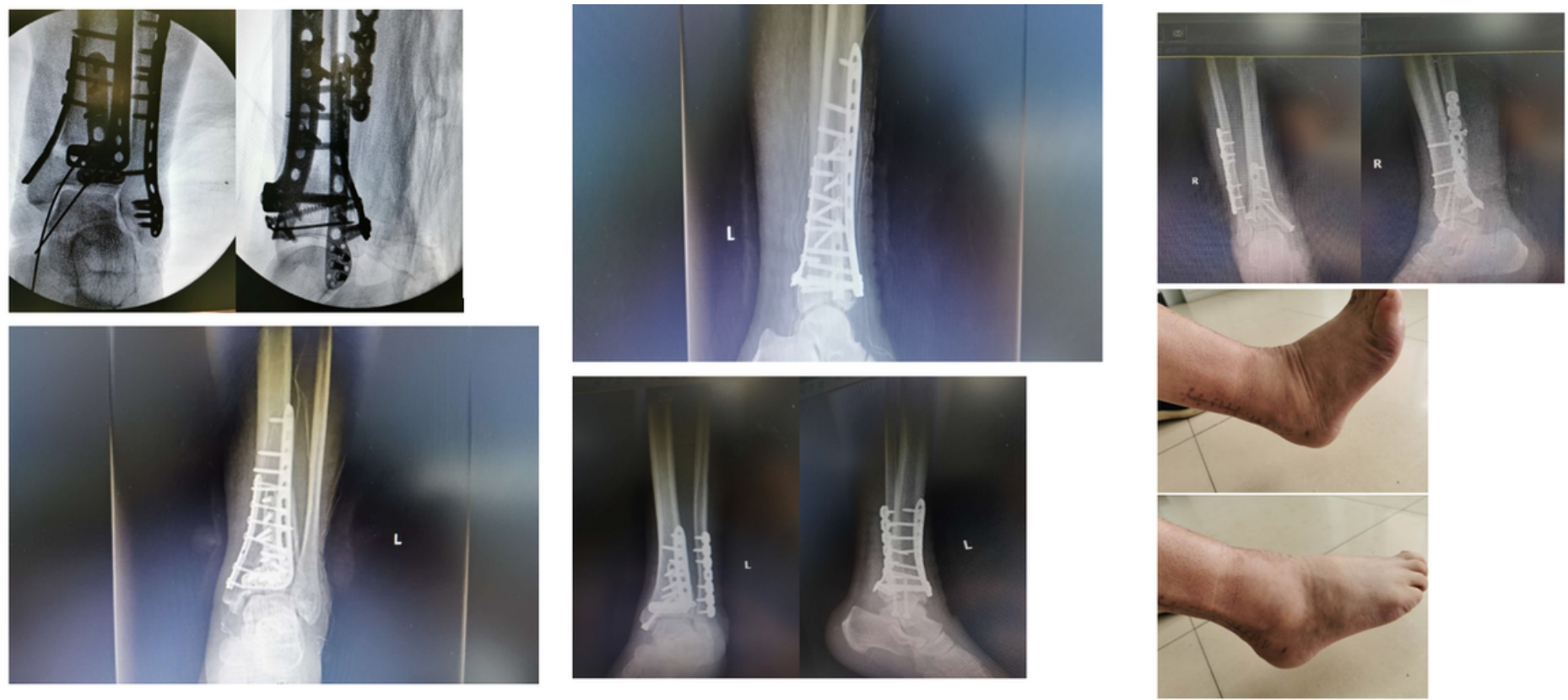

\section{Figure 1}

AOFAS ankle-hindfoot score and Burwell-Charley Radiology Score were compared between two groups. The weight-bearing time after operation of the observation groups 6.91 weeks, which compared favorably to the control groups. Of the twenty-six patients completing outcome questionnaires, all had returned to full weight bearing with 4 having pain with weight bearing in the control group and 1 having pain with 